\title{
Time Dependent Convection and the Pulsations of Polaris
}

\author{
Siobahn M. Morgan ${ }^{1}$, Arthur N. Cox ${ }^{2}$ \\ ${ }^{1}$ University of Northern Iowa, U. S. A., ${ }^{2}$ Los Alamos National Laboratory, U. S. A.
}

The low amplitude radially pulsating classical Cepheid Polaris ( $\alpha$ UMi) has well observed decreasing radial velocity and light amplitudes and an increasing period. Recent work indicates that Polaris may stop pulsating by 1995 (Dinshaw et al, 1989), which would make it an excellent indicator for the red edge of the Cepheid instability strip in the Hertzsprung-Russell diagram.

We have calculated Population I envelope models with 200 mass shells of a $5.5 M_{\odot}$ star near $T_{\text {eff }}=5700 \mathrm{~K}$ and $\log \left(L / L_{\odot}\right)=3.11$ which match Polaris' characteristics (Arellano Ferro, 1984). Models were calculated using the Linear Non Adiabatic pulsation analysis code of Castor (1971). The effects of convection on the pulsational stability were calculated with the time dependent convection theory of Cox, Brownlee and Eilers (1966) as discussed by Cox and Guili (1968). The characteristics of the models were altered until the appropriate period, temperature and luminosity were obtained.

Without including the effects of time dependent convection, we can not find models which are stable against pulsation and which have Polaris' physical characteristics. Using the linear time dependent convection theory we obtain models which are stable against pulsation at temperatures cooler than the red edge. The effect of the mixing length in the standard convection theory on the red edge of the Cepheid instability strip is examined and values for the mixing length of $l=2.2 H_{p}$ are required to obtain models with the period, luminosity and temperature of Polaris. Such values for the mixing length are not unusual considering the values needed for solar models and for Mira variables. Also recent work of the shape on the Cepheid instability region appear to require values of the mixing length close to $2.0 H_{p}$ for stars with masses similar to Polaris' (Morgan, 1992).

\section{References:}

Arellano Ferro, A. 1984, M. N. R. A. S., 209, 481.

Castor, J. I. 1971, Astrophys. J., 166, 109.

Cox, A. N., Brownlee, R. R. and Eilers, D. D. 1966, Astrophys. J., 144, 1024.

Cox, J. P. and Giuli, R. T. 1968, Principles of Stellar Structure, Gordon and Breach, New York, p. 1045.

Dinshaw, N., Matthews, J. M., Walker, G. A. H., and Hill, G. M. 1989, Astronom. J., 98, 2249.

Morgan, S. M. 1992, these proceedings. 